\title{
Diagnostic and Laparoscopic Management of Unicornuate Uterus with Rudimentary Horn
}

\author{
Els van Doorn*, Rainer Kimmig, Bahriye Aktas \\ Department of Obstetrics and Gynecology, University of Essen, Essen, Germany \\ Email: ${ }^{*}$ dea.van.doorn@hotmail.com
}

Received 18 April 2014; revised 17 May 2014; accepted 15 June 2014

Copyright @ 2014 by authors and Scientific Research Publishing Inc.

This work is licensed under the Creative Commons Attribution International License (CC BY).

http://creativecommons.org/licenses/by/4.0/

(c) (i) Open Access

\begin{abstract}
We describe here a case report of a female patient with a unicornuate uterus with noncommunicating left rudimentary horn. The patient presented herself to us due to persistent lower abdominal pains, primarily dysmenorrhea and suspected internal genital endometriosis. Further to additional diagnosis and imaging by vaginal and abdominal ultrasound and abdominal MRI, a suspected rare congenital malformation of the genital tract was established. A normal vagina with cervical system without pathological findings was presented during the operation. The diagnostic hysteroscopy and laparoscopy indicated an intact right unicornuate uterus with a regular tube and ovary. A left rudimentary horn, noncommunicating with cervix and vagina was also presented, also together with a regular tube and ovary. The chromopertubation carried out proved the patency of the right tube. The decision was taken intraoperatively to remove the left rudimentary horn with ipsilateral tube. The surgery could be carried out without complications. We are reporting a patient with persistent lower abdominal pain, who desires to have children, to conceive with a unicornuate uterus and left rudimentary horn with retrograde menstruation and proliferative endometrium. Surgical minimal invasive treatment by laparoscopically removing of the left rudimentary horn and tube took place.
\end{abstract}

\section{Keywords}

Unicornuate Uterus, Rudimentary Horn, Diagnostic and Laparoscopic Management, Endometriosis, Retrograde Menstruation

\footnotetext{
${ }^{*}$ Corresponding author.
} 


\section{Introduction}

This paper reports on the case of a female patient with a unicornuate uterus and left rudimentary horn with retrograde menstruation and proliferative endometrium, who is willing to conceive. She also suffers from a persistent lower abdominal pain. We removed the left rudimentary horn and tube using minimal invasive laparoscopy. We describe this case report, because it is a rare type of mullerian duct malformation and in our opinion, it has educational and scientific merits.

\section{Case Report}

The 25-year-old patient, 0 Gravida, presented herself to us due to a bicornuate uterus for further examination and treatment. The patient had no medical history, except a case history of primary dysmenorrhea. The external MRI carried out showed an indication of unicollis bicornuate uterus, with a left rudimentary horn and suspected non-communication with the cervix and vagina. Clinically there was a strong suspicion of retrograde menstruation with greater fluidity in the Douglas pouch post-menstrually. Normal diagnosis in the area of both kidneys was shown. The MRI was carried out externally due to an existing family history of abnormalities of the uterus (Figure 1).

The gynecological examination revealed a regularly-formed vagina with an unremarkable cervix. Sonographically the MRI exam could also clearly indicate that the rudimentary horn with hematometra certainly bordered the right of the horn. The ovaries appeared to be normal.

Due to the high perimenstrual psychological stress, with the suspected retrograde menstruation of the left uterine horn, the exploratory surgery and treatment were discussed with the patient.

Due to surgical procedure a normal outer genitals, both vagina and cervix was seen. The laparoscopy confirmed normal conditions in the upper abdomen. The following condition was found in the pelvic region: approx. $50 \mathrm{ml}$ blood in the Douglas pouch, due to the retrograde menstruation of the left uterine horn. The right horn appeared to be completely normal with a regular right tube exit, together with normal arrangement of the right ovary. The left side revealed a rudimentary horn, which was only connected to the right horn by one muscular bridge (Figure 2). Here together with a normal left tube exit, which was somewhat elongated, with normal ovarian system. Clinically, isolated endometriosis in the right fossa ovarica region was also strongly suspected, which could be completely removed. Otherwise, no further foci were visible macroscopically.

Simultaneously with the laparoscopy a hysteroscopy was carried out to definitively rule out a connection with both horns. The right uterine cavity showed a normal endometrium and a tubal ostium, with no connection to the left horn. The chromopertubation revealed a prompt patency of the right tube.

Based on the given configuration with safe distance from the ureters on both sides, together with the absence of vessel malformation, the decision was taken with the certain retrograde menstruation through the left tube. The gradual electrocoagulation and free preparation of the tube from the ovary to the left uterine horn was followed by the electrocoagulation of the left rotundum ligaments and peritoneal opening, ventral and dorsal free preparation. The bipolar coagulation of the ovarii proprium ligaments subsequently followed, together with their relocation. Following the resection of the muscular connections the left horn could subsequently be disconnected

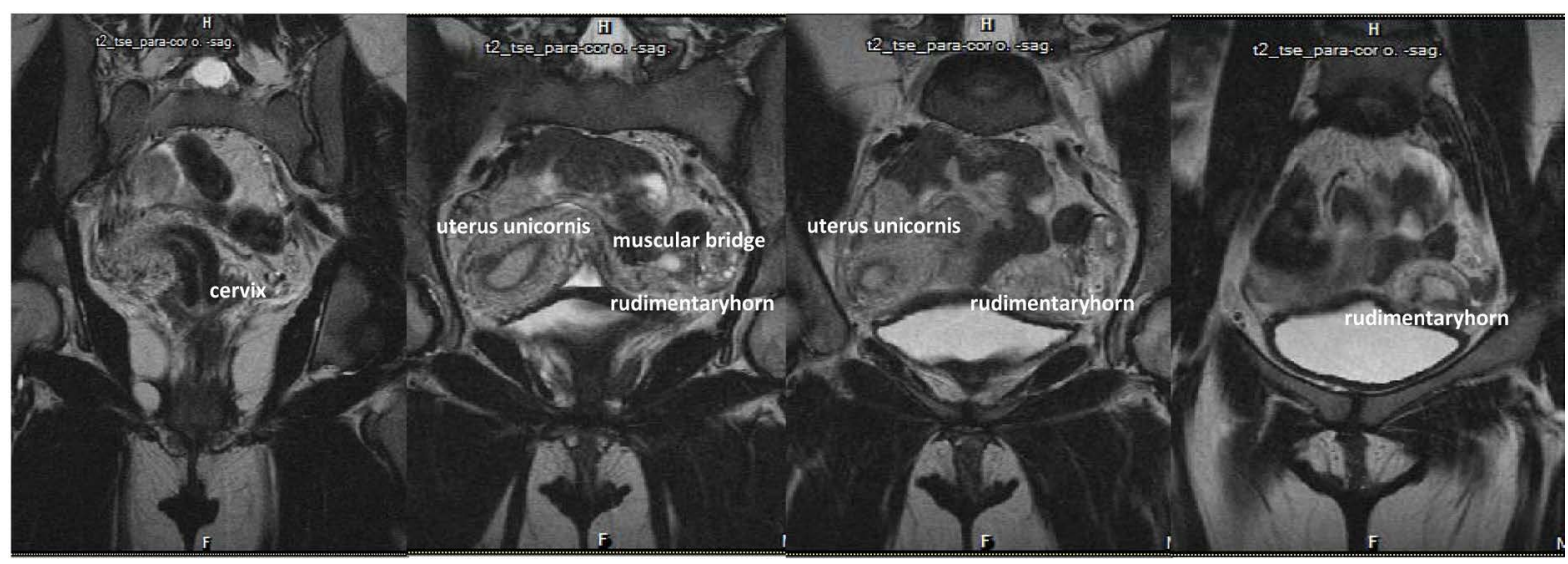

Figure 1. MRI: Uterus unicornis with rudimentary horn. 


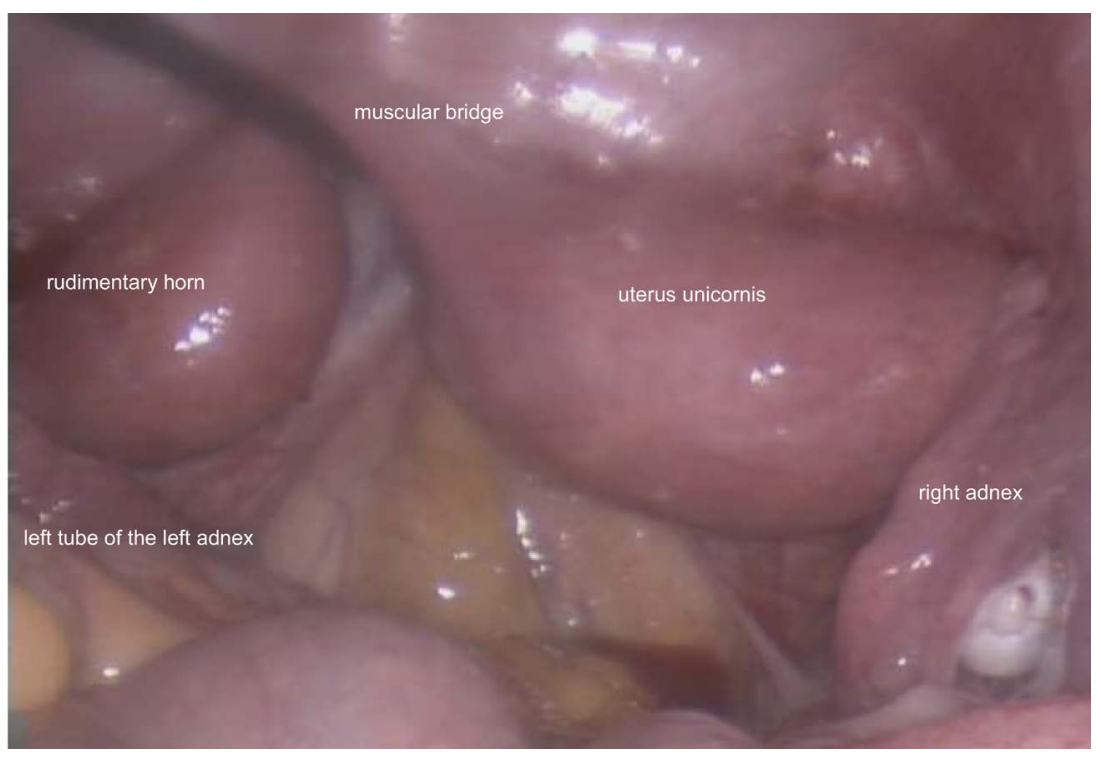

Figure 2. Laparoscopy: unicornuate uterus with rudimentary horn.

from the right horn. In doing so a vessel connection appeared in this muscular connection. Then followed by peritonealising of the wound area to prevent adhesive disease. The final hysteroscopy also revealed a cervix and right uterine horn in normal condition. The postoperative progress was free of complications and the patient could be discharged after 3 postoperative days.

The histology of the resection on the right adnexal mass indicated focal endometriosis. The left resectate consists of a rudimentary uterine horn with proliferative endometrium, regular tube with fimbriae without any suspected malignity.

At the follow up after hospital discharge the patient showed no pain and had no gynecological symptoms and was using her contraception.

\section{Discussion}

Uterine malformations are described in the literature with a prevalence on average of $0.2 \%$ to $0.4 \%$. The uterus develops physiologically as the caudal sections of the Müllerian ducts fuse together. Uterine malformations are commonly associated with vaginal and cervical anomalies, together with malformations of the adnexa and the urogenital tracts, and indicate embryological development disorders [1] [2].

These anomalies are usually due to abortion, premature births and anomalies with the position of the fetus. The prevalence in infertile patients is therefore up to around 13\% [2].

The most common clinical manifestations of unicornuate uterus with non-comunicating rudimentary horn, are dysmenorrhea, abdominal pain and hematometra in the non-communicating rudimentary horn [3].

Guiding principles are family history, (3D)-ultrasound or ultrasound with infusion of the uterine cavity, hysterosalpingography, MRI, hysteroscopy together with laparoscopy and if necessary with chromopertubation [1] [2] [4].

In 1907 Strassman carried out the first systematic classification of uterine anomalies. A second classification exercise was undertaken by Buttram in 1979, which was revised by the American Fertility Society in 1988. This highlighted a further deficit in treatment as a result of which the VCUAM classification was developed. This classification focuses on anatomical variations in female external and internal genitals. The following sub-categories are distinguished: Vagina (V), Cervix (C), Uterus (U), Adnex (A). Associated malformations commonly occur with malformations of the Müllerian ducts (M) [2] [4].

The aim of this classification is to provide a clearly defined description of a malformation taking into consideration anatomical circumstances and embryogenesis.

Our patient with unicornuate uterus and left rudimentary horn is classified as follows:

VCUAM U4a, A0 ML.

In the present unicornuate uterus with a lateral fusion defect, one horn had developed normally whilst the 
other horn had developed rudimentarily (74\%). After the present dates the rudimentary horn, with a partially functional endometrium, did not communicate with the other horn to the degree of $70 \%-90 \%$. At $40 \%$, this deformity has the highest incidence of renal malformations. An MRI is recommended in all cases. An invasive examination should succeed if a surgical correction appears worthwhile [2] [5].

Diagnostic examination and laparoscopic management of these uterine anomalies are seldom described, therefore with this case report we wish to advance a contribution to literature which enhances the attention paid to minimal invasive surgical possibilities and to prevent more hematometra, extrauterine gravidity etc.

Individual cases of hematometra, endometriosis, infertility, extrauterine gravidity and gravidity in rudimentary horn are described, in which various surgical procedures were successful [5]-[8].

One case report described a tubal pregnancy on the side of the noncommunicating rudimentary horn with the ipsilateral ovary carrying a corpus luteum in a unicornuate uterus. In that case also indicated that transperitoneal migration of the sperm or ovum occurs around the impregnating period [9] [10].

\section{Conclusions}

With concern to our case report, the decision to take out the rudimentary horn with ipsilateral tube was right. In literature, there are no studies specified towards conservative treatment or minimal invasive laparoscopically resection of the rudimentary horn with ipsilateral tube. The main key to successful management of the rudimentary horn is early detection and preventive surgery in our opinion.

The living birth rates for unicornuate uterus are quoted as $27 \%$ - $40 \%$ [4].

\section{Conflicts of Interest}

The patient was treated by the doctors of duty Bahriye Aktas and Els van Doorn. There are no conflicts of interest, according to this case report.

\section{Consent}

Consent of the patient was given.

\section{References}

[1] Kiechle, M., Beinder, E., Bosinski, H.A.G., Brucker, C., Bühren, C. and Chaoui, R. (2007) Gynäkologie und Geburtshilfe. Elsevier GmbH, Urban \& Fischer Verlag, 33-35.

[2] Geibel, D. and Rimbach, S. (2013) Uterine Fehlbildungen. ÄP Gynäkologie, 2, 16-21.

[3] Poel, G., Aggarwal, A., Devi, K., Takkar, N., Saha, P.K. and Huria, A. (2005) Unicornuate Uterus with Non-Communicating Rudimentary Horn-Different Clinical Presentations. Journal of Obstetrics and Gynaecology of India, 55, 155-158.

[4] (2010) Leitlinie der Deutschen Gesellschaft für Gynäkologie und Geburtshilfe (DGGG): S1-Leitlinie Gynäkologie 015/052. Weibliche Genitale Fehlbildungen.

[5] Liatsikos, S.A., Tsikouras, P., Souftas, V., Ammari, A., Prassopoulos, P., et al. (2010) Diagnosis and Laparoscopic Management of Rudimentary Uterine Horn in a Teenage Girl, Presenting with Haematometra and Severe Endometriosis: Our Experience and Review of Literature. Minimally Invasive Therapy and Allied Technologies, 19, $241-247$. http://dx.doi.org/10.3109/13645701003644491

[6] Fuchs, F., Guillot, E., Cordier, A.-G., Chis, C., Raynal, P. and Panel, P. (2008) Rupture of Non-Communicating Rudimentary Pregnant Uterine Horn in a Pseudo-Unicornuate Uterus at 23 Weeks of Amenorrhea: Case Report. Elsevier, 400-402.

[7] Daskalakis, G., Pilalis, A., Lykeridou, K. and Antsaklis, A. (2002) Rupture of Non-Communicating Rudimentary Uterine Horn Pregnancy. Obstetrics Gynecology, 100, 1108-1110. http://dx.doi.org/10.1016/S0029-7844(02)02153-1

[8] Lennox, G., Pantazi, S., Keunen, J., Van Mieghem, T. and Allen, L. (2013) Minimally Invasive Surgical Management of a Second Trimester Pregnancy in a Rudimentary Uterine Horn. Journal of Obstetrics and Gynaecology Canada, 35, 468-472.

[9] Handa, Y., Hoshi, N., Yamada, H., Wada, S., Kudo, M., Tsuda, K., et al. (1999) Tubal Pregnancy in a Unicornuate Uterus with Rudimentary Horn: A Case Report. American Society for Reproductive Medicine, Elsevier, 72, 354-356.

[10] Jayasinghe, Y., Rane, A., Stalewski, H. and Grover, S. (2005) The Presentation and Early Diagnosis of the Rudimentary Uterine Horn: Review. American College of Obstetricans and Gynecologists, 105, 1456-1467. 
Scientific Research Publishing (SCIRP) is one of the largest Open Access journal publishers. It is currently publishing more than 200 open access, online, peer-reviewed journals covering a wide range of academic disciplines. SCIRP serves the worldwide academic communities and contributes to the progress and application of science with its publication.

Other selected journals from SCIRP are listed as below. Submit your manuscript to us via either submit@scirp.org or Online Submission Portal.
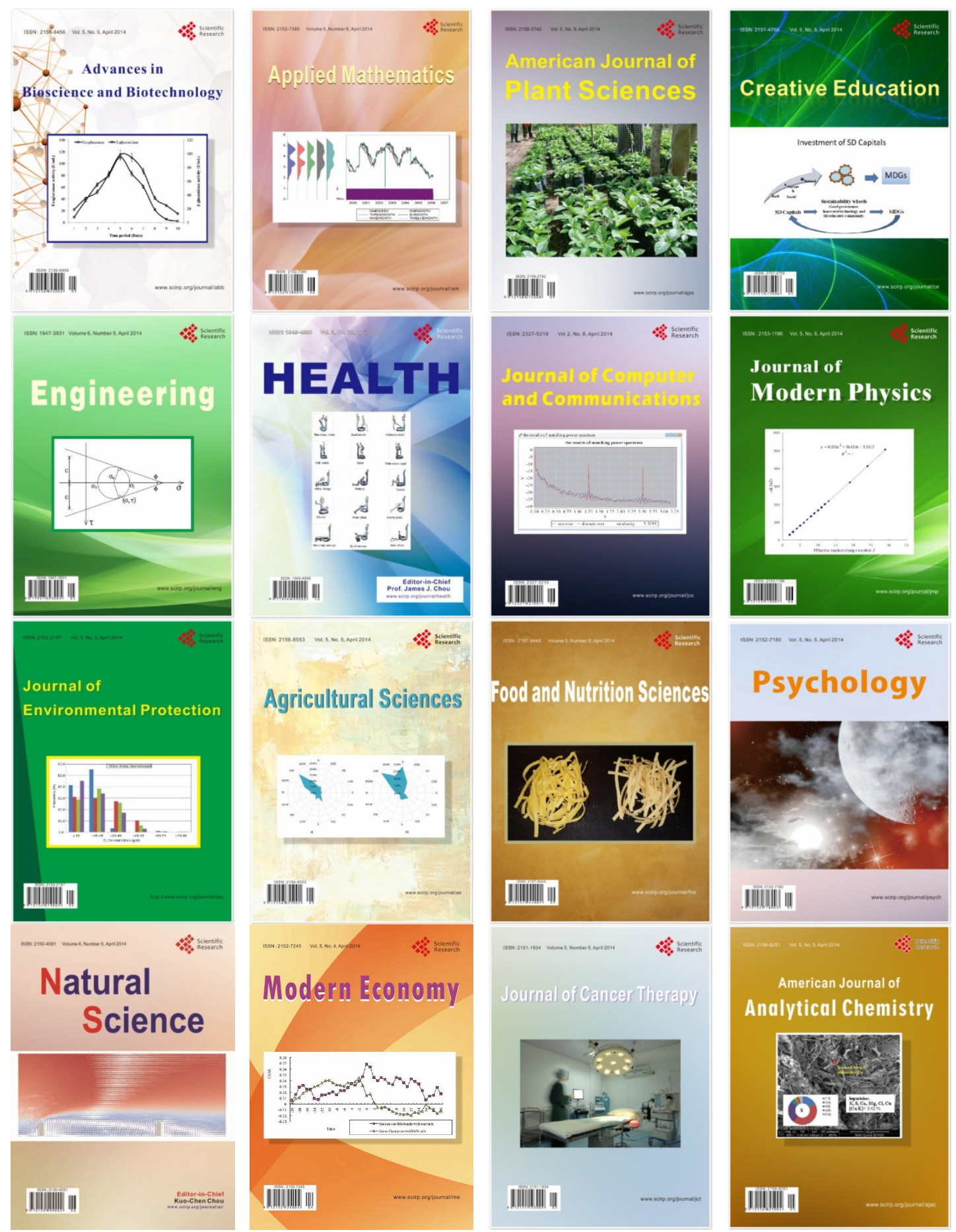